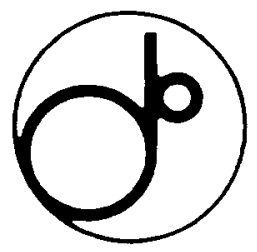

KEK Preprint 95-182

December 1995

H/D

\title{
Performance Analysis of a Switch-type Event Builder with Global Traffic Control System
}

Y. NAGASAKA, M. NOMACHI, O. SASAKI

and

M. TAIRADATE

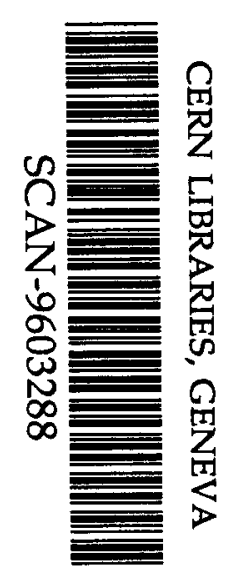

$\operatorname{sig} 9613$

Submitted to the Real Time 95 Conference, East Lansing, MI, U.S.A., May 23 - 26, 1995, and will be published in the IEEE Transactions on Nuclear Science. 
National Laboratory for High Energy Physics, 1995

KEK Reports are available from:

Technical Information \& Library

National Laboratory for High Energy Physics

1-1 Oho, Tsukuba-shi

Ibaraki-ken, 305

JAPAN

Phone: 0298-64-1171

Telex: $\quad 3652-534$ (Domestic)

(0)3652-534 (International)

Fax: $\quad$ 0298-64-4604

Cable: $\quad$ KEK OHO

E-mail: LIBRARY@JPNKEKVX (Bitnet Address)

library@kekvax.kek.jp (Internet Address) 


\title{
Performance analysis of a switch-type event builder with global traffic control system
}

\author{
Yasushi NAGASAKA, Masaharu NOMACHI and Osamu SASAKI \\ National Laboratory for High Energy Physics \\ Oho 1-1, Tsukuba, Ibaraki 305, Japan \\ Masaru TAIRADATE \\ Graduate University for Advanced Studies \\ Oho 1-1, Tsukuba 305, Japan
}

\begin{abstract}
Switch type event builders have been proposed to handle large amounts of data traffic. A global traffic control system is one of the efficient ways to utilize a switch for event builders. The performance of a switch-type event builder with global traffic control is analyzed in this report.
\end{abstract}

\section{INTRODUCTION}

Switch-type event builders have been studied for high-speed data acquisition systems [1-6]. Event fragments from the data sources are collected at the processing elements through the switch. Switch modules developed for network systems have been considered as a candidate for switch-type event builders. Most of the network switch modules are designed to handle random data traffic. Congestion may happen statistically if the random data traffic becomes heavy. However, for event builders, the switch modules have to handle coherent data traffic. In an event builder, event fragments from all sources are concentrated into one destination. Such data flow causes congestion in a switching network or at destination nodes even for little data traffic. Such congestion may suppress the effective bandwidth of the switching network [7].

The congestion of coherent traffic can be avoided by shaping the traffic to have a random character. This method has been intensively studied in the RD31 group at CERN $[3,4]$. Another way to avoid congestion of coherent traffic is to establish a global traffic control. A circuit switch was applied to an event builder at Fermilab $[1,2]$. The data traffic is controlled by a global traffic signal. The aim of this paper is to analyze the performance of a switch-type event builder with a global traffic control.

\section{GLOBAL TRAFFIC CONTROL}

\section{A. Control scheme}

Data traffic in an event builder is analogous to traffic at a crossroads. A traffic or a set of signals may control traffic so as to avoid collisions. It is an example of a global control system on a crossroads. A set of traffic paths is switched to another one while the streams are stopped. A policeman may control the traffic according to the amount of incoming traffic. He uses real-time traffic information at each moment. It may be a very efficient way to control the traffic if he does a good job. However, in a complicated crossroads, it will be difficult for him to find the best way to control.

A set of traffic signals can also be used to control the traffic. A set of signal lights is sequentially changed to another set. The sequence is defined a priori according to the estimated amount of incoming traffic. It does not respond to a burst of traffic. However, it may be good enough for most cases if the sequence is correctly determined.

The event builder with a global traffic control system that we analyzed is analogous to a traffic control system with a set of traffic signals. A set of traffic signals corresponds to a switch configuration. The switch configurations are sequentially selected by a global control signal. The sequence and duration of each switch configuration are assigned by using the estimated incoming data rates. It does not require a central controller that may cause a bottle-neck of the system. A global control system is a simple control scheme but its performance as an event builder should be studied.

Data are transferred according to the following sequences. Each source node has one queue for each destination. The event fragments to be sent to that destination are placed at the queue. Each destination node has one queue for each data source. The event fragments coming from that source are placed at the queue. There are virtual circuits between queues at the source nodes and queues at the output nodes. This situation is shown in Figure 1. A set of connections is selected by a global control signal. The duration of each switch configuration is assigned according to the estimation of the incoming data rates. Data are transferred as packets. The size of packets is determined by the duration of the set. If the packet size is larger than average event fragment size, the packet may contain several event fragments to be sent to the destination. If the packet 
size is smaller than the average event fragment size, an event is transferred with several packets.

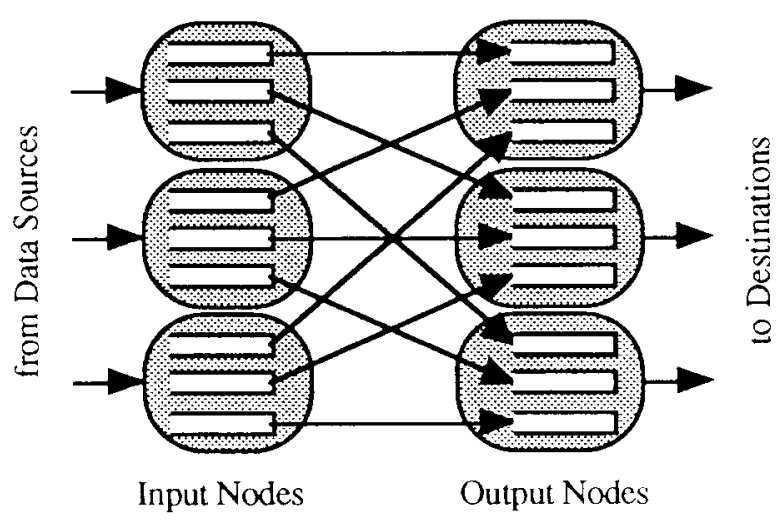

Figure 1. Logical connections between sources and destinations.

Queue occupancy of a queue on a source node can be analyzed by the characteristics of an input data stream and the characteristics of pre-defined data extraction sequence from the input queue. A simple analysis based on queuing theory can be used to analyze the system in some simple conditions.

\section{B. Barrel shifter}

A barrel shifter is one of the simple switch configuration sequences. For example, Figure 2 shows a sequence of a barrel shifter. If the sizes of out-going data streams are balanced, equal time slots for each configuration may be assigned equally. Such an event builder system has been developed at Fermilab $[1,2]$. More complicated assignments may be needed to handle unbalanced data streams. We have analyzed the performance of an event builder with a barrel shifter switch in this report. Performance is analyzed as a function of input load, a switching frequency and a switch size.

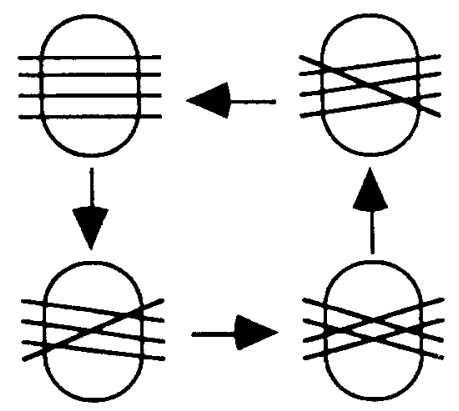

Figure 2. Sequence of barrel shifter switch configurations.

\section{Model}

We have assumed the following conditions:

- The event interval of successive events has an exponential distribution;

- Event fragment size has an exponential distribution;

- There is no correlation among the size of event fragments;

- Destinations are assigned cyclically according to the event number

The cycle has " $k$ " destinations. Here, " $k$ " is the number of destinations. An event is directed to one particular destination once every " $k$ " events. An interval of two successive events directed to the destination has a distribution of the convolution of " $k$ " independent exponential distributions. It is known as a k-Erlang distribution. We have analyzed for a case of a large " $k$ ", or for a large event builder. An Erlang distribution can be approximated by a delta function in that case. An event arrival is deterministic if the interval has a distribution of a delta function. This approximation is applied to the analysis.

Characteristics of the source queues have been calculated in reference [8]. In this report, they are reanalyzed with queuing theory. Simulation results are compared with the calculations. The simulation is written in C language with a user-written simple scheduler routine. The simulation is done for a simplified system that has only one input queue on each source node. Events are generated with a deterministic interval. Event fragments are extracted sequentially as a packet to the output queue in the destination node. An event is extracted when all event fragments are collected on the output queues on the destination node.

The system latency is an important characteristic of an event builder and affects data throughput. Queue latency has a simple relation to the queue length. The relation is known as Little's formula [9].

$$
\text { Queue length }=\text { Latency } \cdot \text { Event rate }
$$

A longer queue length may cause a buffer overflow. The number of event fragments in a queue is analyzed to evaluate the system performance.

\section{ANALYSIS}

\section{A. Load dependence}

The number of event fragments in the input queue is analyzed as a function of traffic intensity which is the ratio of the input data rate over the maximum rate 
that the switch can handle. A switch can handle up to the traffic intensity of "1.0" if the queue has infinite length. However, a large queue occupancy may cause a long system latency. With the finite length of queues in the system, a high traffic intensity may cause a queue overflow.

In the small packet limit, the queue occupancy is easily calculated. Data extraction time is proportional to the event fragment size, which has an exponential distribution. It is a "service time" in the queuing theory. The interval of an event arrival on the queue is deterministic. Therefore, the system characteristics are described by the $\mathrm{D} / \mathrm{M} / 1$ model in the queuing theory. The queue length $(\mathrm{L})$, which is the number of event fragments in a queue, is calculated as the following equation [10].

$$
\text { traffic intensity }=L \cdot\left(1-e^{-1 / L}\right)
$$

Figure 3 shows the results of the calculation and the simulation. The system works up to high traffic intensity.
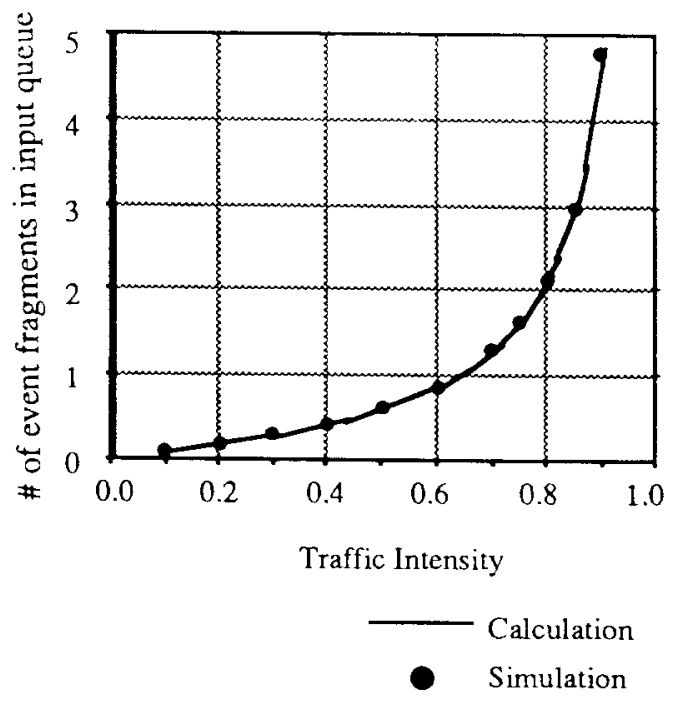

Figure 3. Load dependence for small packet case.

\section{B. Switching frequency dependence}

The packet size dependence of the queue length is analyzed. It is determined by the switching frequency. The number of event fragments in the input queue is analyzed for various packet sizes. The result of the analysis and the simulation are shown in Figure 4 for the traffic intensity of " 0.4 ". Simulation results are shown as circles. Calculations are done for two extreme cases. In the small packet case, the number of event fragments is calculated as 0.45 traffic intensity by the $D / M / 1$ model as discussed in the preceding section. The number of event fragments in the input queue is independent of the packet size as far as the approximation is applicable.
In the large packet size case, the number of event fragments just before the data extraction from the queue should be the average number of event fragments in a packet multiplied by the traffic intensity if traffic is low. It comes from the definition of the traffic intensity. The average queue length (L) over an interval of time is a half of that maximum queue length because the queue length increases linearly from zero to the maximum in the interval.

$$
L=\frac{1}{2} \cdot \text { traffic intensity } \cdot \text { packet size }
$$

Two lines in the figure show the results of the calculations on the extreme cases. In the case of the packet size being nearly equal to the average event fragment size, the simulation results interpolate the calculations for the extreme cases well.

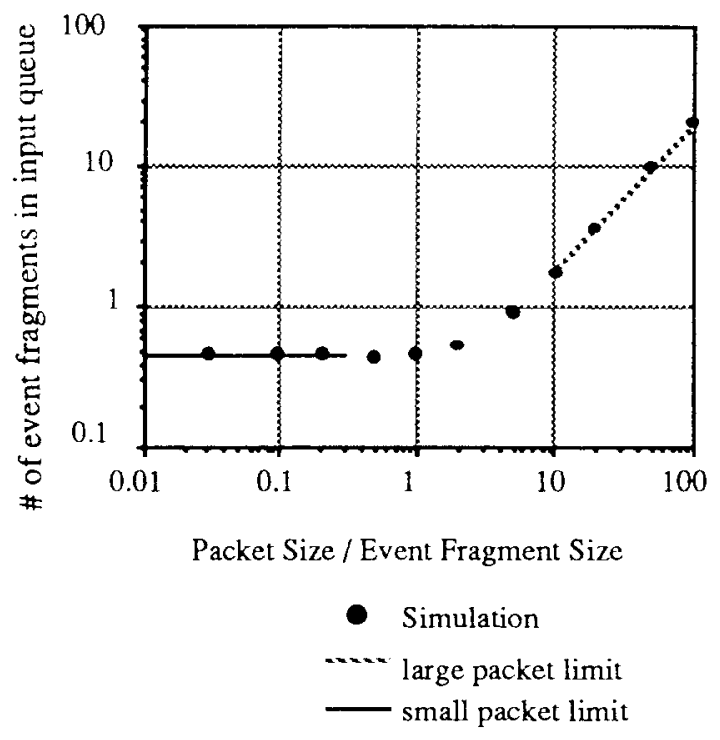

Figure 4. Packet size dependence.

A large packet size causes a longer queue length. Smaller packet sizes are better for getting shorter latency. However, a packet size smaller than the event fragment does not help to reduce the queue length. The smaller packet size means more frequent switching. It may cause more overhead time that reduces data throughput and increases the queue length. Therefore, it is better not to have an unnecessary high switching frequency. If the switching overhead time is negligible compared to the data transfer time of one event fragment, the packet size should be the same as the event fragment size to obtain a minimum latency. In other words, the frequency of switching should be less than the maximum event rate that the switch can handle. 


\section{Switch size dependence}

The number of event fragments in an output queue is analyzed as a function of the switch size. The number of event fragments in the input queue is independent of switch size. It is determined only by traffic intensity and packet size as shown in the preceding sections. However, the number of event fragments in the output queue may depend on the switch size. The output queues must hold event fragments until all event fragments of that event are collected at the destination node. In the large packet size case, only one switching cycle is needed to collect all event fragments. It does not depend on the switch size. However, if the packet size is smaller than the event fragment size, the completion of data transfer of that event must wait for the arrival of the largest event fragment. It depends on the switch size.

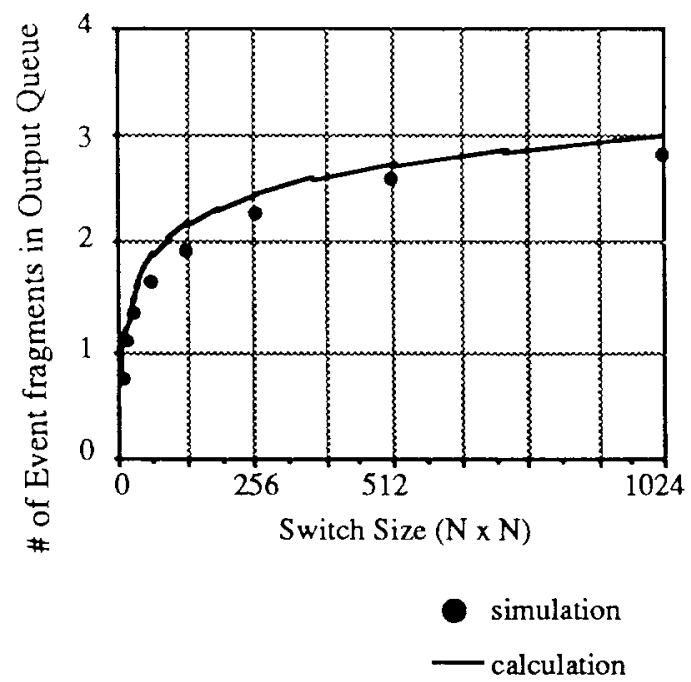

Figure 5. Switch size dependence for small packet case.

The output queues must hold event fragments until the largest event fragment is transferred. During that period, the other output queues may receive the following event fragments. If the largest fragment size is " $r$ " times larger than the average one, the other output queues may receive " $r$ traffic intensity" event fragments while waiting. The average of the largest event fragment size is calculated in the appendix. The result is shown in equation 4.

$$
\frac{<\text { Largest fragment size }>}{<\text { Average fragment size }>}=\sum_{k=1}^{\text {switch size }} \frac{1}{k}
$$

The average number of event fragments in the output queue can be determined by the traffic intensity. The calculation and simulation results are shown in Figure 5. There is a small discrepancy between the calculation and the simulation, but the general tendency is the same. The large switch size causes a longer queue length. It increases logarithmically as a function of switch size.

\section{SUMMARY}

The performance of a switch-type event builder with global traffic control is analyzed. It is shown that a simple control system works well as an event builder. We could show that it is not necessary that the switching frequency to be so high to achieve low latency. And it is almost scalable to a large system.

The results of the calculation and the simulation are in good agreement. Characteristics of a switchtype event builder with a global traffic control are predictable in an analytical way based on queuing theory.

\section{APPENDIX}

\section{The distribution of largest event fragment size}

A probability density function of the event fragment size is defined as $f(x)$.

$$
f(x)=\frac{l}{v} e^{-x / v}
$$

Here, " $v "$ is the average size of event fragments. Size of the largest event fragment among the $n$-event fragments is:

$$
X_{n}=\max \left(x_{1}, x_{2}, \ldots \ldots \ldots \ldots x_{n}\right)
$$

A probability density function of a variable $X_{n}$ is defined as $F_{n}\left(X_{n}\right)$. A variable $X_{n+1}$ is the larger of $X_{n}$ and $x_{n+1}$.

$$
X_{n+1}=\max \left(X_{n} x_{n+1}\right)
$$

The probability that the variable $X_{n+1}$ has a value of $x$ is a sum of the two probabilities. One probability is that the variable $X_{n}$ has a value of $x$ and the variable $x_{n+1}$ is smaller than $x$. The other probability is that the variable $x_{n+1}$ has a value $x$ and the variable $X_{n}$ is smaller than $x$.

$$
F_{n+1}(x)=\int_{0}^{x} f(x) F_{n}(y) d y+\int_{0}^{x} f(y) F_{n}(x) d y
$$

Equation (8) is solved analytically:

$$
F_{n}(x)=\frac{n}{v} \cdot e^{-x / v}\left(1-e^{-x / v}\right)^{n-1}
$$


An expectation value is obtained as shown in equation 10 .

$$
<X_{n}>=\int_{0}^{\infty} x \cdot F_{n}(x) d x=v \cdot \sum_{i=1}^{n} \frac{1}{i}
$$

\section{REFERENCES}

[1] Ed. Barsotti et al., A Proposed Scalable Parallel Open Architecture Data Acquisition System for Low to High Rate Experiments, Test Beam and All SSC Detectors, IEEE Trans. NS, NS-37 No3.(1990)

[2] D.Black , M.Bawden et al., Results From a Data Acquisition System Prototype Project Using a SwitchBased Event Builder,1991 IEEE Nucl. Science Symposium

[3] M. Letheren et al., An Asynchronous Data-Driven EventBuilding Scheme based on ATM Switching Fabrics, IEEE trans. on Nuclear Science, Vol. 41, No.1, Feb. 1994.

[4] M. Costa et al., NEBULAS - A high performance datadriven event building architecture based on an asynchronous self-routing packet-switching network (RD31 Status report '95), CERN/LHCC/95-14, March (1995)

[5] B.Greiman, A scalable Fibre Channel Architecture for Event Building, International Data Acquisition Conference, October 1994.

[6] M. Nomachi, Other research projects (Fermilab / LBL / KEK), presentation at International Data Acquisition Conference, Fermilab, October 1994.

[7] Yasushi NAGASAKA, Masaharu NOMACHI, Osamu SASAKI, Atsushi MANABE, Stability of coherent data traffic in a switching network, contribution to ninth conference on real-time computer applications in nuclear, particle and plasma physics, East-Lansing, 1995

[8] Masaharu Nomachi, Event builder queue occupancy, SDC-93-566, (1993), SSCL.

[9] J.D.C.Little, A proof of the queuing formula: $L=\lambda W$, Opns. Res. 9(3), (1961),383-387

[10] It will be found on many textbooks on the queuing theory, for example, T.L.Saaty, Elements of queuing theory with applications, McGraw-hill, (1961) 
